\title{
Background and First Results from the New AAO/UKST H $\alpha$ Survey
}

\author{
Quentin A. Parker ${ }^{1}$ and Steven Phillipps ${ }^{2}$ \\ 1 Anglo-Australian Observatory, Siding Spring Mountain, NSW 2357, Australia \\ qap@aaocbn.aao.gov.au \\ 2 Astrophysics Group, University of Bristol, Tyndall Avenue, Bristol BS8 1TL, England \\ Received 1997 September 22, accepted 1997 November 20
}

\begin{abstract}
The UK Schmidt Telescope (UKST) of the Anglo-Australian Observatory (AAO) has just embarked on a new $\mathrm{H} \alpha$ survey of the Southern Galactic Plane, Magellanic Clouds and selected regions using a specially designed, high-specification, monolithic, interference filter. It is probably the largest of its kind for astronomy. It is being used in combination with Kodak Tech Pan film-based emulsion. This emulsion not only has a useful sensitivity peak at $\mathrm{H} \alpha$ but also possesses extremely fine grain and an exceptionally high DQE for the hypersensitised product $(\sim 10 \%)$. This leads to excellent imaging, sensitivity and low noise. It is clear that CCDs cannot yet match the wide-area coverage, uniformity and resolution of the UKST/Tech Pan combination for undertaking such a survey. The survey will initially include about 233 Galactic Plane and 40 Magellanic Cloud fields on 4-degree centres and will take about 3 years to complete. Some preliminary images from the new survey are presented and compared with the best previously available from the UKST. Examples of the first survey discoveries, new resolved Galactic planetary nebulae, are also given. This survey will have an unprecedented combination of area coverage, depth and resolution, superior to those of any previous optical survey of ionised gas in the Galaxy. Many new discoveries and research avenues are expected.
\end{abstract}

Keywords: instrumentation: detectors - techniques: interferometric - surveys stars: formation - ISM - HII regions

\section{Need for the Survey}

Despite the importance of star formation in our own Galaxy, its variation between galaxies and the need to understand the resultant gaseous emission line structures visible on a wide range of angular scales, little survey work has been undertaken in a form that combines both large area coverage, high sensitivity and good resolution. The nearest starforming complexes may lie as close as 100 parsecs with sizes of tens of parsecs. Such structures often subtend angular sizes of a degree or more yet exhibit fine detail at the arcsecond level. To study the interaction of these ionised structures with their large-scale environment we need surveys of considerable extent at good angular resolution. Most work to date has concentrated on relatively small regions for specific study at high spatial or velocity resolution (e.g. Russeil et al. 1998, present issue p. 9) or very large areas at low resolution (e.g. Rodgers, Campbell \& Whiteoak 1960; Kogure et al. 1982; and more recently Buxton et al. and Dennison et al.- see present issue pp. 24 and 147). It is only now that surveys are beginning to simultaneously tackle issues of coverage, sensitivity and resolution in either a spatial sense (our survey) or in velocity space (e.g. Reynolds et al. 1998, present issue p. 14). There is a clear need for a high angular resolution optical survey to complement studies at other wavebands.

The only existing UKST wide-area $\mathrm{H} \alpha$ survey work dates to the late 1970s (e.g. Davies, Elliot \& Meaburn 1976) using mainly coarse-grained (though fast) 098-04 emulsion and a far from optimum filter. Many parts of the Galactic plane are unsurveyed at decent resolutions, particularly the outer extensions beyond a few degrees from the Galactic equator. The northern Milky Way above Dec. $=-20^{\circ}$ has not been covered at all. Progress in other wavebands highlights the urgent need for an optical counterpart for the detailed study of Galactic gas. Table 1 gives a list of the various $\mathrm{H} \alpha$ surveys currently under way and known to the authors, together with the KYOTO survey (Kogure et al. 1982) as an example from the older photographic work. Our new survey will clearly contribute much to the detailed investigation of star formation and the general ISM in terms of its coverage and resolution.

\section{Tech Pan Film as Detector for UKST H $\alpha$ Survey}

The successful implementation of high-resolution, panchromatic Tech Pan film on the UKST, coupled with its peak sensitivity at $\mathrm{H} \alpha$, was a prime motivation behind the new Galactic Plane survey. Details of the many advantages offered by this remarkable emulsion 
Table 1. Basic characteristics of various current $\mathrm{H} \alpha$ surveys

\begin{tabular}{lrrclclc}
\hline \multicolumn{1}{c}{ Survey } & $\begin{array}{c}\text { Area } \\
\left(\text { degree }^{2}\right)\end{array}$ & $\begin{array}{c}\text { Resolution } \\
(\operatorname{arcsec})\end{array}$ & $\begin{array}{c}\text { Sensitivity* } \\
(\mathrm{R})\end{array}$ & $\begin{array}{c}\text { FOV } \\
(\text { degree })\end{array}$ & $\begin{array}{c}\text { Finish } \\
\text { date }\end{array}$ & $\begin{array}{c}\text { Telescope/detector } \\
\text { Filter }\end{array}$ & $\begin{array}{c}\text { FWHM }(\AA) \\
\text { FW })\end{array}$ \\
\hline AAO/UKST & 4000 & 1 & $5-10 ?$ & $5 \cdot 5 \times 5 \cdot 5$ & 1999 & UKST, Tech Pan film & 70 \\
WHAM $^{1}$ & 30000 & 3600 & $0 \cdot 25$ & 1 & 1998 & $0 \cdot 6 \mathrm{~m}$, Fabry-Perot, CCD & $0 \cdot 25$ \\
MSSSO $^{2}$ & 4000 & 12 & $?$ & $7 \times 7$ & 1998 & $400 \mathrm{~mm} \mathrm{~F} / 4 \cdot 5,2 \mathrm{~K}$ CCD & 55 \\
MARSEILLE $^{3}$ & 280 & 9 & $0 \cdot 2$ & $0 \cdot 6 \times 0 \cdot 6$ & 1999 & $36 \mathrm{~cm}$, Fabry-Perot & $5 \mathrm{~km} / \mathrm{s}$ \\
VIRGINIA $^{4}$ & 4000 & 96 & $0 \cdot 3$ & $10 \times 10$ & $?$ & $\mathrm{f} / 1 \cdot 2$ lens, CCD & 17 \\
MICHIGAN $^{5}$ & 100 & 3 & 20 & $1 \cdot 1 \times 1 \cdot 1$ & 1999 & Curtis Schmidt, 2k CCD & 30 \\
KYOTO $^{6}$ & 4000 & 30 & $\sim 10$ & 17 & 1982 & $14 \cdot 3 \mathrm{~cm}+103 \mathrm{aE}$ & 280 \\
\hline
\end{tabular}

${ }^{1}$ Reynolds et al.; ${ }^{2}$ Buxton et al.; ${ }^{3}$ Russeil et al.; ${ }^{4}$ Dennison et al.; ${ }^{5}$ Smith et al. (all in this present issue); ${ }^{6}$ Kogure et al. (1982). ${ }^{*}$ There is a lack of consistent units for measuring $\mathrm{H} \alpha$ sensitivity levels (emission measures), which are given in either Rayleighs $\left(\mathrm{R}, \mathrm{cm}^{-6} / \mathrm{pc}\right)$ or $\mathrm{erg} / \mathrm{cm}^{2} / \mathrm{s} / \mathrm{arc}^{2}$. The conversion factors are $0 \cdot 3 \mathrm{R}=1 \mathrm{~cm}^{-6} / \mathrm{pc}=2 \times 10^{-18} \mathrm{erg} / \mathrm{cm}^{2} / \mathrm{s} / \mathrm{arc}^{2}$.

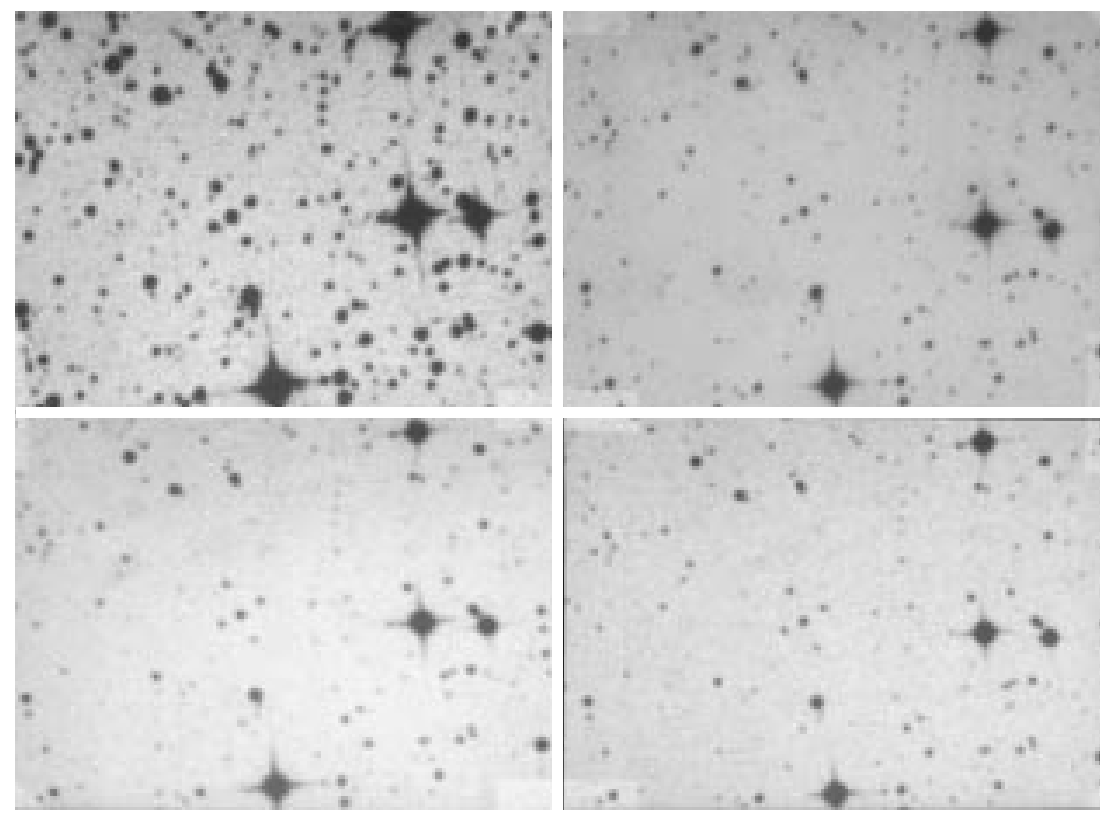

Figure 1-Direct UKST image comparisons between a standard R-band IIIaF exposure (top left), Tech Pan 2 hour H $\alpha$ exposure (top right), short-red (bottom left) and I survey (bottom right).

for general wide-field photographic imaging can be found in Parker et al. (1995, 1998), whilst Malin (1998, present issue p. 38) also describes its suitablity for further contrast enhancement. Generally speaking we have found that in good seeing, Tech Pan UKST $\mathrm{R}$ band exposures go about 1 magnitude deeper than the equivalent standard IIIaF R-band images with improved imaging, resolution and lower noise characteristics. Comparisons of IIIaF and Tech Pan emulsion sensitivities as a function of wavelength through a $100 \AA \mathrm{H} \alpha$ filter were obtained with a calibration spectrograph. The results convinced us that there was no speed/sensitivity/reciprocity penalty in adopting Tech Pan for use with a narrowband $\mathrm{H} \alpha$ filter cf. IIIaF. It is an ideal wide-field photographic detector for use with an $\mathrm{H} \alpha$ filter. The associated paper by Masheder, Phillipps \& Parker (1998, present issue p. 5) details the emulsion's specific scientific advantages for undertaking the $\mathrm{H} \alpha$ survey.

Tech Pan's superiority can also be seen in Figure 1, a set of $4 \cdot 4 \times 3$ arcminute identical sky regions from near the centres of four UKST exposures of standard Galactic Plane survey field 213. The top left image is from the A-grade survey $\mathrm{R}$ plate taken with IIIaF emulsion and the OG590 R-band filter with 63 minutes exposure. It has a visual point-source detection limit of $R \sim 21 \cdot 5$. The top right image is from the equivalent exposure taken with Tech Pan and the new $\mathrm{H} \alpha$ filter with 120 minutes exposure. The bottom left is the short-red A-grade survey plate taken with 098-04 fast but coarser-grained emulsion with a narrower 630 red filter $(600 \AA)$ in 10 minutes exposure. The bottom right image is the A-grade 'I' survey plate taken with IV-N emulsion with a 715 filter and 90 minutes exposure.

Prior to the availability of quantitative $\mathrm{H} \alpha$ data from SuperCOSMOS measuring machine scans of the new films, simple visual examination of these exposures was performed. It is estimated that for point source detection the $\mathrm{H} \alpha 120$ minute Tech Pan exposure goes at least 0.5 magnitudes deeper than the short-red and is about 1 magnitude less deep than the $\mathrm{R}$ survey but has considerably superior 
resolution. It is also about 0.5 magnitude deeper than the I survey. We are not yet sky-limited with the 2 hour $\mathrm{H} \alpha$ exposure.

\section{High-quality $\mathrm{H} \alpha$ Interference Filter}

As no suitable AAO $\mathrm{H} \alpha$ filter was currently available, a vendor (Barr Associates) was identified to supply a high-specification, large monolithic interference filter for use at the UKST's focal surface. Very stringent optical tolerances were necessary as the filter was to be used in the converging $\mathrm{f} / 2.48$ beam and the excellent imaging capabilities of Tech Pan must not be compromised. Details of the specifications achieved are given in Parker \& Bland-Hawthorn (1998, present issue p. 33). This interference filter, probably the largest of its type in astronomy, has a multilayer dielectric stack with a 'circular aperture' of $305 \mathrm{~mm}$ diameter coated on to the $356 \times 356$ mm RG610 glass substrate. This permits a field of about 5.5 degrees diameter to be observed in $\mathrm{H} \alpha$. The CSIRO National Measurement Laboratory in Sydney has quantitatively confirmed that the filter meets the stringent optical specifications set, especially the $70 \AA$ FWHM filter bandwidth and $6590 \AA$ central wavelength in collimated light.

The exceptional optical quality of the filter has been demonstrated on the telescope by the first 2 hour $\mathrm{H} \alpha$ exposures taken in good seeing during April 1997. These preliminary exposures exhibit excellent imaging and uniformity across the entire field. They also confirm the $305 \mathrm{~mm}$ diameter clear aperture of excellent $\mathrm{H} \alpha$ sensitivity of about $5 \cdot 5$ degrees on the sky.

\section{Survey Planning and Logistics}

The new survey, timely in respect of telescope loading, commenced in April 1997. Unfortunately the filter's $5 \cdot 5$ degree diameter field does not provide contiguous $\mathrm{H} \alpha$ sky coverage when set on standard 5 degree field centres. This is due to the 1.5 degree overlap between standard UKST $6 \cdot 5 \times 6 \cdot 5$ degree fields. A small $(\sim 1)$ degree area in the overlap regions is missed. A conservative 4 degree field centre separation was adopted which ensures no gaps in $\mathrm{H} \alpha$ coverage. About 233 such fields are needed to cover the Southern Galactic Plane. This will then be extended to the outer regions of the Galactic Plane and to declinations from +0 to +15 degrees. Exposures will be of the order of 3 hours and the initial survey region will take about 3 years to complete. Although not sky-limited, the 3 hour exposure times are a good compromise between survey progress and exposure quality, due to the increased likelihood of seeing variations, cloud interruptions and effects of field rotation. The narrow-band nature of the $\mathrm{H} \alpha$ filter means that the survey could continue in good seeing grey/bright time when the sky is too bright for normal observations. The photometric integrity of the survey is currently being assessed via independent narrow-band CCD photometry from the Curtis Schmidt at CTIO and with reference to previously studied objects over a range of UKST fields.

\section{Survey Accessibility}

To maximise the availability and usefulness of the new survey, the AAO, Schmidt Telescope Panel and $\mathrm{H} \alpha$ survey consortium have upgraded the Galactic Plane/Magellanic Clouds proposal to a full AAO survey. This will ensure rigorous quality control of the survey and its timely accessibility to the community. The consortium also intend to produce a fully calibrated digitised survey database of $10 \mu \mathrm{m}$ resolution pixel data. This will be released to the astronomical community as a CD-ROM atlas from SuperCOSMOS scans as soon as is practicable. The CD-ROM atlas may be released in installments for faster community access. Film copies of the survey should also be available.

Up-to-date information on survey progress, examples of preliminary images, filter specifications, details of the $\mathrm{H} \alpha$ survey consortium membership and other pertinent details can be found on the WWW at: http://www.aao.gov.au/local/www/qap/halpha_ pages.html.

This survey should complement the radio maps from the ATNF and MOST, those of the new Parkes $\mathrm{H}$ i multibeam survey as well as those from mm-wave telescopes here and overseas. The prospects for collaboration and comparison from studies in other wavebands are considered excellent.

\section{Preliminary $\mathrm{H} \alpha$ Image Comparisons}

An impression of the significant information gains from the new Tech $\mathrm{Pan} \mathrm{H} \alpha$ filter combination compared to previous UKST $\mathrm{H} \alpha$ imaging is presented in Figure 2. The images show an identical $6 \times 5$ arcminute area in an interesting LMC emission bubble from four different UKST $\mathrm{H} \alpha$ exposures taken with a range of filters/emulsions and exposure times. The top left image is data from a 2 hour Tech Pan exposure with the new filter; at top right is an 80 minute equivalent (same emulsion and filter). There is a dramatic increase in information in the longer exposure with no degradation in image quality. The bottom left image gives a 90 minute exposure through the old $120 \AA$ FWHM AAO656 $254 \times 254$ $\mathrm{mm} \mathrm{H} \alpha$ filter with the fast, coarse-grained 098-04 emulsion. This is the worst-performing combination for tracing/discovering the fine detailed structure visible in the new exposures. Finally, at bottom right is a 3 hour exposure taken with the same AAO656 filter with the standard IIIaF red-sensitive 


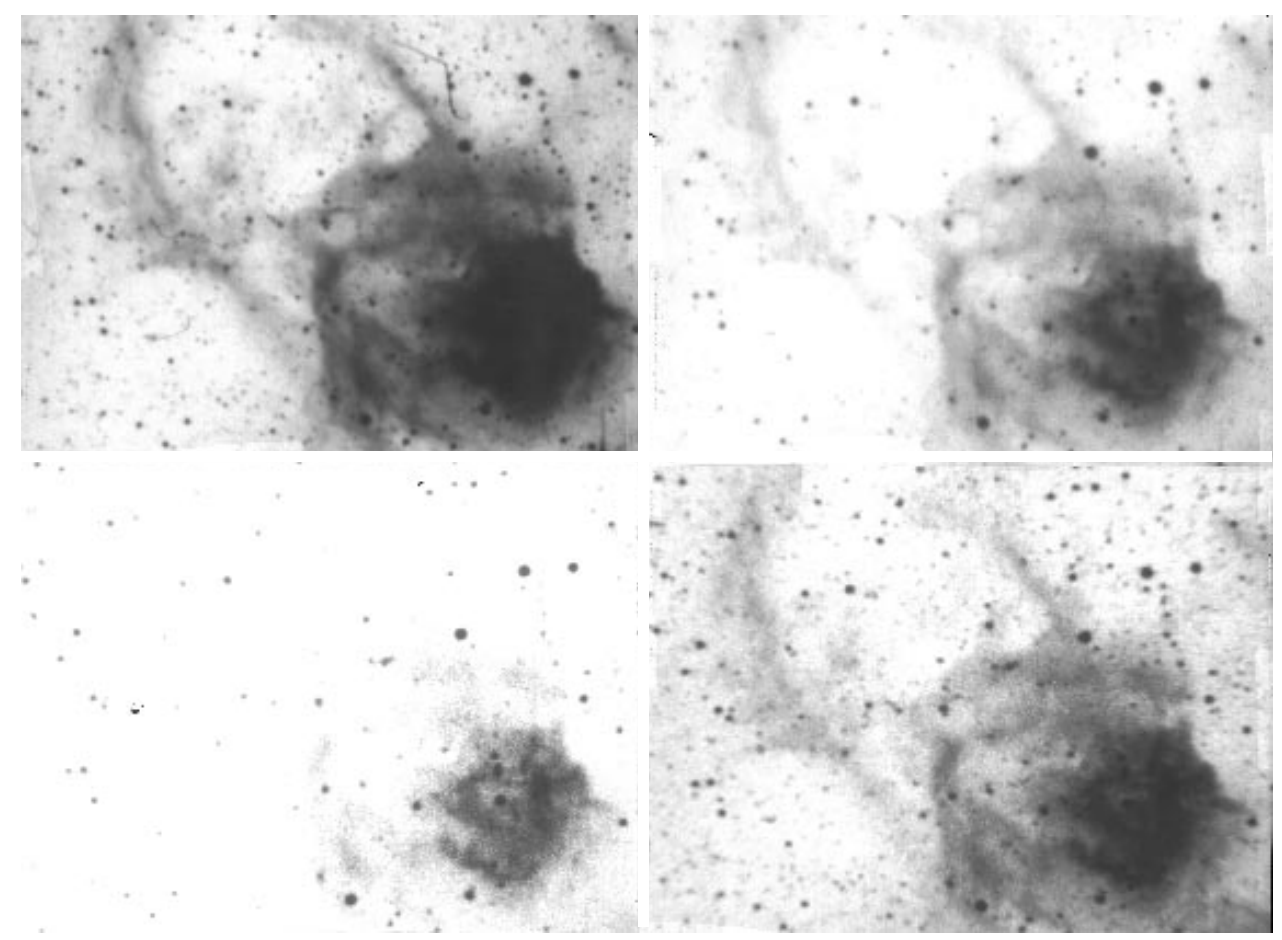

Figure 2-Comparison of image quality/depth of the new $\mathrm{H} \alpha$ Tech Pan combination filter with equivalent UKST images taken with a range of other $\mathrm{H} \alpha$ filters, emulsions and exposure times. See Section 6 for further details.

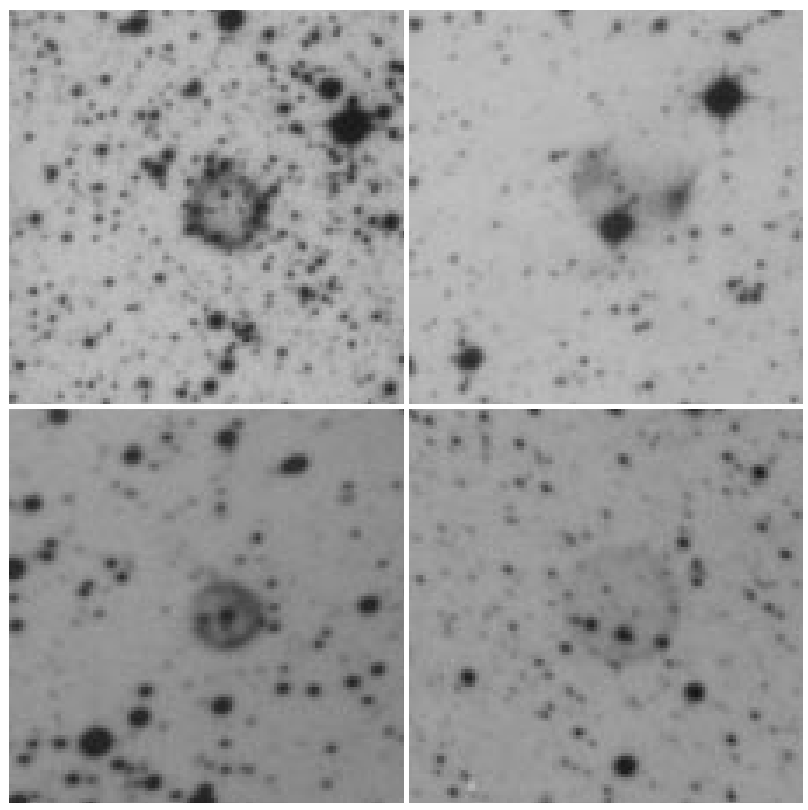

Figure 3-Four new PNe discovered on 3 hour $\mathrm{H} \alpha$ survey exposures. The top left and right PNe were discovered on exposure HA17614 (field 334). The bottom left PNe is from HA17617 (field 226) and the bottom right from HA17619 (field 392). Each plot is $2 \cdot 6 \times 2 \cdot 6$ arcminutes in size.

emulsion. Despite the wider passband and longer exposure, the depth and detail of the Tech Pan new filter equivalent in Figure 3 (top left) is far superior.

\section{First Discoveries from $\mathrm{H} \alpha$ Survey}

As an example of the impact that this survey can have for our study of the Galaxy, we present in
Figure 3 four examples of new, resolved planetary nebulae $(\mathrm{PNe})$. They were discovered by one of us (QAP) from simple visual scrutiny of some of the first 3 hour $\mathrm{H} \alpha$ exposures. They were confirmed as new discoveries by cross-checking with SIMBAD and the online Strasbourg-ESO catalogue of Planetary Nebulae (Acker et al. 1992) and the Acker supplement (Acker et al. 1996). These new 
PNe are invisible or barely detectable on previous deep standard R-band exposures of the field but are easily found on the new $\mathrm{H} \alpha$ exposures. Follow-up visual searches of ten new 3 hour $\mathrm{H} \alpha$ exposures by QAP and M. Hartley have found about 30 new $\mathrm{PNe}$ candidates. If this frequency is replicated throughout the survey then about 450 new optically detected/discovered resolved PNe can be expected. This can be compared with the $\sim 1500$ currently known in the Galaxy (both hemispheres) based on entries in the Acker catalogues. Simple visual scanning of the survey films could significantly increase the number of Southern Galactic Plane PNe. Planned SuperCOSMOS scans of these films would undoubtedly pick up many more unresolved or barely resolved candidates for further study via comparison with the equivalent 'continuum' shortred exposures which have similar (slightly shallower) depth.

\section{Acknowledgments}

It is a pleasure to thank the AAO for its foresight in agreeing to purchase the $\mathrm{H} \alpha$ filter which has made this survey possible. We are grateful for the support of the staff of the UKST and especially M. Hartley for providing an independent visual scanning check of the new PN discoveries reported here. We also thank M. Hartley and J. Bland-Hawthorn for useful discussions and the helpful comments of the anonymous referee. QAP is currently on special leave from the Royal Observatory Edinburgh.

Acker, A., Marcout, J., \& Ochsenbein, F. 1996, First supplement to the Strasbourg-ESO Catalogue of Galactic Planetary Nebulae (Observatoire de Strasbourg)

Acker, A., Ochsenbein, F., Stenholm, B., Marcout, J., \& Schohn, C. 1992, Strasbourg-ESO Catalogue of Galactic Planetary Nebulae, ISBN 3-923524-41-2

Buxton, M., Bessell, M., \& Watson, B. 1998, PASA, 15, 24

Davies, R. D., Elliot, K. H., \& Meaburn, J. 1976, Mem. RAS, 81,89

Dennison, B., Simonetti, J. H., \& Topasna, G. A. 1998, PASA, 15, 147

Kogure, T., Kobayashi, Y., Sasaki, T., Sakka, K., Miyajima, K., \& Nakano, M. 1982, Contribution from the Department of Astronomy, University of Kyoto, No. 133, ISSN 03880230

Malin, D. F. 1998, PASA, 15, 38

Masheder, M. R. W., Phillipps, S., \& Parker, Q. A. 1998, PASA, 15, 5

Parker, Q. A., \& Bland-Hawthorn, J. 1998, PASA, 15, 33

Parker, Q. A., Phillipps, S., Malin, D. F., Cannon, R., \& Russell, K. S. 1998, MNRAS, submitted

Parker, Q. A., Phillipps, S., \& Morgan, D. H. 1995, IAU Colloq. 148, ASP Conf. Ser. 84, ed. J. M. Chapman et al. (San Francisco: ASP), p. 96

Reynolds, R. J., Tufte, S. L., Haffner, L. M., Jaehnig, K., \& Percival, J. W. 1998, PASA, 15, 14

Rodgers, A. W., Campbell, C. T., \& Whiteoak, J. B. 1960, MNRAS, 121, 103

Russeil, D., Georgelin, Y. M., Amram, P., Georgelin, Y. P., Laval, A., \& Marcelin, M. 1998, PASA, 15, 9 